\title{
DYNAMICS OF A HIGH DENSITY ION-BEAM WITH ELECTRON COOLING IN HIMAC SYNCHROTRON
}

\author{
T. Uesugi*, T. Fujisawa, K. Noda, D. Tann, NIRS, Chiba, 263-8555 JAPAN, \\ I. Meshkov, E. Syresin, JINR, Dubna, 141980 RUSSIA, \\ S. Shibuya, H. Uchiyama, AEC, Chiba, JAPAN, \\ Y. Hashimoto, KEK, Tsukuba, JAPAN, S. Ninomiya, RCNP, Osaka, JAPAN
}

\section{Abstract}

High density circulating-ion beam was obtained with cool-stacking injection in the HIMAC synchrotron. The ion density was saturated at $\sim 0.9 \times 10^{7}$ ions $/ \mathrm{mm}^{2}$. Coherent transverse instability was observed when ion- and electronbeam density was high. The dynamics of the cooled ionbeam are described in this report.

\section{INTRODUCTION}

The heavy ion medical accelerator in Chiba [1] (HI$\mathrm{MAC})$ is the accelerator complex constructed in national institute of radiological sciences (NIRS) for cancer therapy and other researches. Since 2000, electron cooling (EC) experiments have been carried out at the synchrotron in order to develop new technologies in heavy-ion therapy and related fields. One of the objectives of the HIMAC cooler is to increase the beam intensity of heavier ions, such as Fe, for risk estimations under low-dose exposure in space [2].

Such a high density ion-beam includes strong spacecharge effects. In the HIMAC synchrotron, the dynamics of a cooled high-intensity beam was investigated using coasting beams of $\mathrm{Ar}^{18+}$. Cool-stacking injection was conducted in order to increase the beam intensity. Beams were successively injected as shown in Fig. 1 using the horizontal space which was created by EC. Stacked intensity at $K$ 'th injection is given by

$$
N_{K}=N_{\text {inj }} \frac{1-\exp \left(-K T_{\mathrm{inj}} / \tau\right)}{1-\exp \left(-T_{\mathrm{inj}} / \tau\right)},
$$

where $N_{\text {inj }}$ is the intensity of one-batch injection, $T_{\text {inj }}$ the injection-repetition time, $\tau$ the ion-loss lifetime. Experimental parameters are listed in Table 1.

The transverse ion distributions and their coherent oscillations were non-destructively mesured with gas-sheet beam profile monitor (SBPM) [3] and beam position monitor (PON), respectively. The SBPM, at which the betatron amplitude function is $\left(\beta_{x}, \beta_{y}\right)=(9 \mathrm{~m}, 7 \mathrm{~m})$, can measure the transverse two-dimensional ion-distribution with $0.8 \mathrm{~mm}$ resolution at FWHM.

With coasting beams, the maximum intensity of $2.5 \times$ $10^{9}$ ions were accumulated with cool-stacking injection [5]. In order to obtain such a high-intensity stack, it was essential to minimize the slow ion-losses and to damp the coherent instability, which are described in the following section.

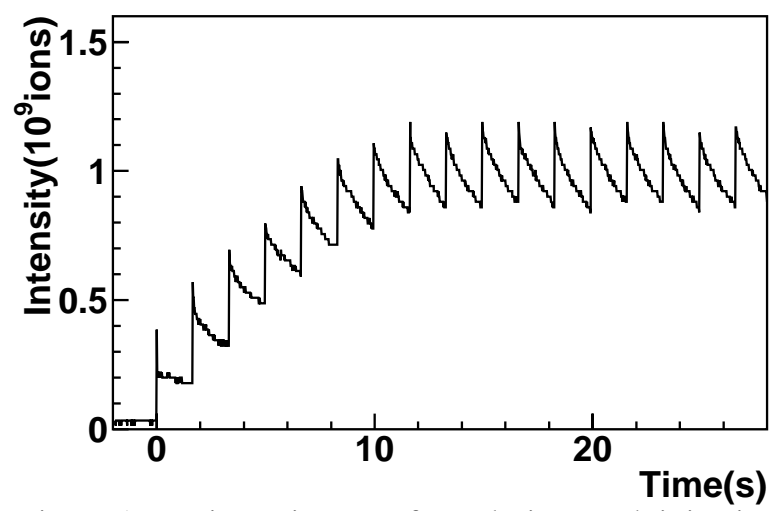

Figure 1: Ion intensity waveform during stack injection.

The limitation on the transverse cooling was also investigated with the beam size measurements with the SBPM. Past experiments in other synchrotrons showed that the limitation came from the intra-beam scattering (IBS), where the ion density in the three dimensional phase-space was approximately limitted [4]. In the HIMAC synchrotron, there was a stronger restriction at the intensity of higher than $\sim 10^{8}$ ions/ring, where the real-space density was limitted at $\sim 0.9 \times 10^{7}$ ions $/ \mathrm{mm}^{2}$.

\section{HIGH INTENSITY ACCUMULATION}

The operation parameters, such as electron-beam current $\left(I_{e}\right)$, magnetic expansion factor $(R)$, injection repetition

Table 1: Experimental conditions

\begin{tabular}{|c|c|}
\hline Parameter & Value \\
\hline \multicolumn{2}{|l|}{ Ring } \\
\hline Circumference & $129.6 \mathrm{~m}$ \\
\hline Particle, energy & ${ }^{40} \mathrm{Ar}^{18+}, 6 \mathrm{MeV} / \mathrm{u}$ \\
\hline Betatron tune & $(3.69 / 3.13)$ \\
\hline & or $(3.69 / 2.89)$ \\
\hline Revolution period & $4 \mu \mathrm{s}$ \\
\hline \multicolumn{2}{|l|}{$\mathrm{EC}$} \\
\hline Current & $25-200 \mathrm{~mA}$ \\
\hline Cathode diameter & $35 \mathrm{~mm}$ \\
\hline Cathode temperature & $100 \mathrm{meV}$ \\
\hline Solenoid field & $0.05 \mathrm{~T}, 1.2 \mathrm{~m}$ \\
\hline Magnetic expansion factor & $R=1.7,2.8,3.3,3.8$ \\
\hline
\end{tabular}




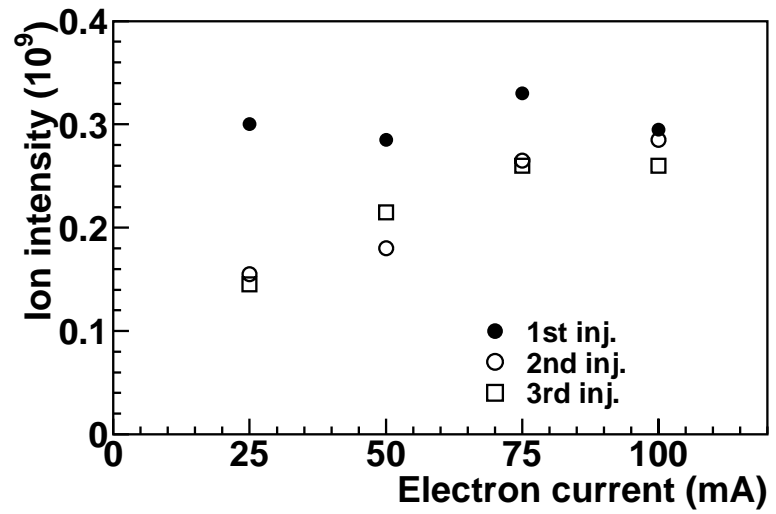

Figure 2: Increase of the ion intensity at the first, the second and the third injections.

period $\left(T_{\mathrm{inj}}\right)$ and bump height, were optimized in coolstacking experiments in order to increase the stacked intensity. As indicated by Eq. (1), the higher stack intensity is obtained when injected number of particles $\left(N_{\text {inj }}\right)$ is increased and/or the beam loss among injections $(\operatorname{Tinj} / \tau)$ is suppressed. Further, the high stack intensity led to coherent instabilities. This section describes how the maximum stack intensity was obtained.

\section{Injection loss}

A part of the ion-beam is lossed by the bump-orbit excitation for the injection, and effectively decreases $N_{\text {inj }}$. The number of the ions to be lost is determined obviously by the relation among the cooling rate $(\lambda)$, the injection repetition time $\left(T_{\mathrm{inj}}\right)$, and the available space between maximum bump orbit and the septum electrode.

Fig. 2 compares the increase of the stacked intensity $\left(N_{\mathrm{inj}}\right)$ at the first, the second and the third injections for different $\mathrm{e}^{-}$-beam currents. The $T_{\text {inj }}$ and the bump height were fixed here. The reduction of the $N_{\text {inj }}$ at the second injection corresponds to the ion beam-loss due to the bump excitation. Fig. 2 shows that the loss became small when the $\mathrm{e}^{-}$-beam current, ie the cooling rate, was high. Maximum stacked intensity was also increased with increasing $\mathrm{e}^{-}$-beam current.

\section{Rapid beam-loss}

Coherent instability occurred at high ion- and $\mathrm{e}^{-}$density, correlated with the dipole-mode transverse oscillation of the ion-beam as the upper diagram of Fig. 3. The instability restricted the high intensity accumulation. The observations of beam profile showed that the direction of the oscillations was inclined in the transverse space at the instability. This fact indicates the $x-y$ coupling of the betatron oscillation. The instability had the maximum growth rate at the difference resonance, $Q_{x}-Q_{y}=1$. The instability was driven by the interaction with ion- and $\mathrm{e}^{-}$-beams $[8,9,10]$.

The instability could be damped by reducing the peak density of the ion-beam by applying transverse rfexcitation at a frequency near to the transverse betatron

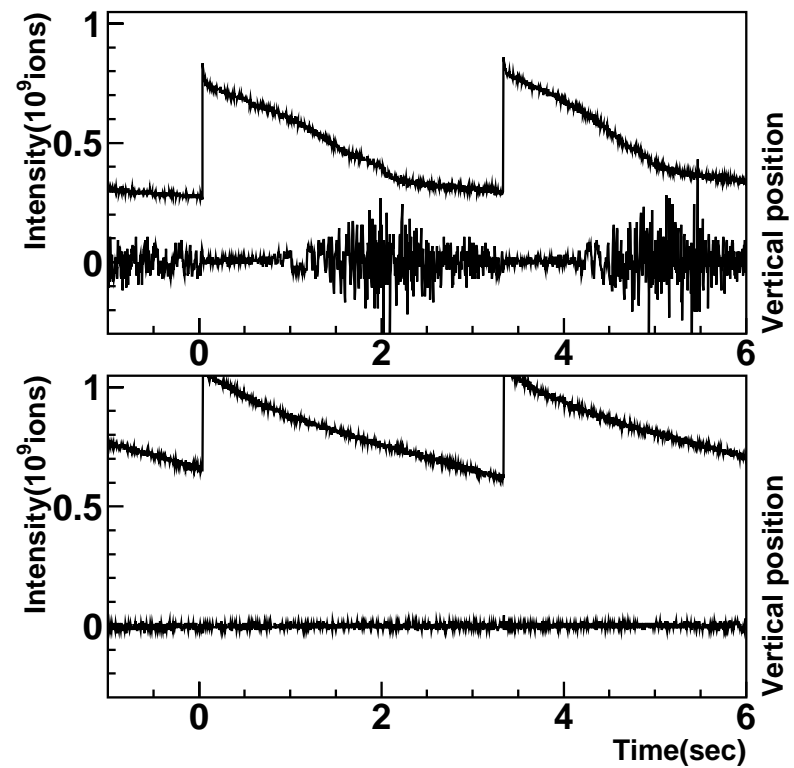

Figure 3: Coherent instability (upper diagram) was damped by applying rf-excitation (lower one). Ion-intensity and vertical oscillations are plotted.

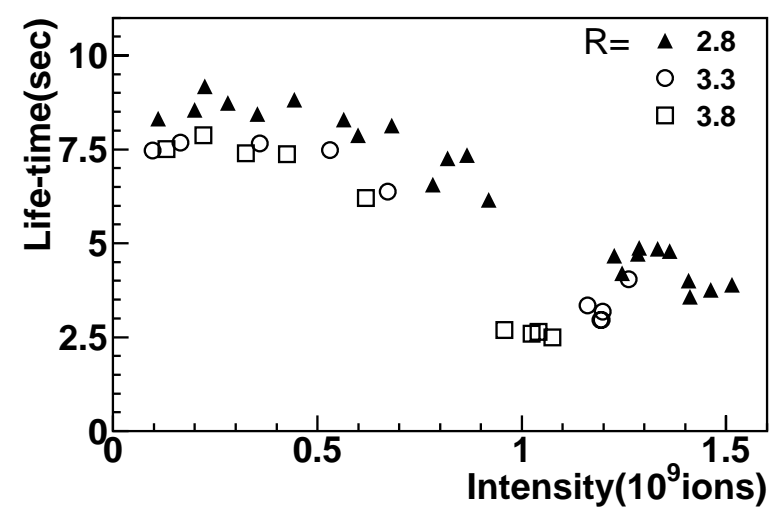

Figure 4: Ion-beam lifetime vs intensity for various expansion factors. The $\mathrm{e}^{-}$-beam current was fixed at $150 \mathrm{~mA}$.

frequency. Fig. 3 shows that the coherent instability was damped and the stack intensity was considerably increased.

\section{Slow beam-loss}

Eq. (1) indicates that the maximum stack intensity is also limitted by the lifetime $(\tau)$. As is seen in Fig. 1, the lifetime of the slow beam-loss was decreased as the intensity became high. Degradation of the vacuum is one of the source of the lifetime reduction at high intensity.

The ion lifetime was measured with different $I_{e}$ and $R$ by analyzing the slow beam-loss waveform after stopping the injection. Figure 4 shows that the lifetime was increased with decreasing $R$ from 3.8 to 2.8 [6]. On the other hand, the dependency of the lifetime on $I_{e}$ was negligible in the wide range of $50 \mathrm{~mA} \sim 175 \mathrm{~mA}$. Therefore the dependence of the lifetime on $R$ is not explained by the cooling rate $(\lambda)$, because, assuming that $\lambda \propto I_{e} / R$, the change of $\lambda$ by $R$ was around $30 \%$, while it was more than $70 \%$ by 


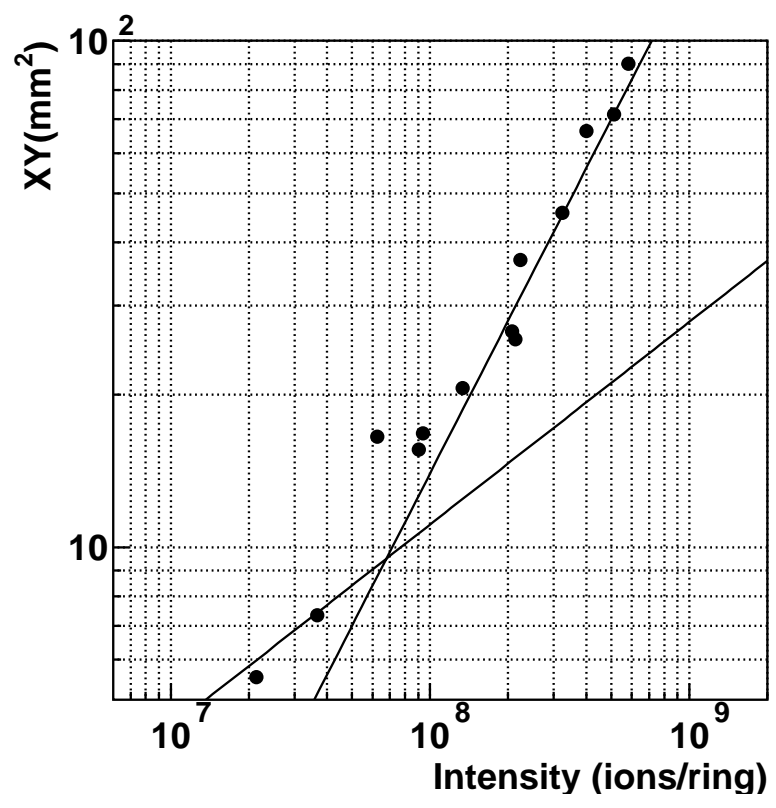

Figure 5: Products of the transverse FWHM beam sizes of cool equilibrium $(X Y)$ at different intensity. Solid lines correspond to $X Y / N=1.1 \times 10^{-7} \mathrm{~mm}^{2}$ and $X Y / N^{2 / 5}=$ $0.017 \mathrm{~mm}^{2}$, respectively.

$I_{e}$. The experimental results indicate that the existence of the electron beam outside of the ion beam contributes to the slow-beam loss.

It seems that the slow-beam loss is related to the diffusion process of the ion-beam inside the $\mathrm{e}^{-}$-beam [7]. The ions with large betatron amplitude is affected the diffusion to be lost. This process strongly depends on the diameter of the $\mathrm{e}^{-}$-beam. However, it should be noted that the diameter of the $\mathrm{e}^{-}$-beam, at the expansion factor of $2.8 \sim 3.8$, corresponds to the ion-beam emittance of larger than the vertical acceptance. Thus, the mechanism of the slow beamloss is not clearly understood, and further investigation is required.

Finally, the maximum intensity of $2.5 \times 10^{9}$ ions were accumulated with the optimum conditions of $T_{\mathrm{inj}}=1.0 \mathrm{~s}$, $I_{e}=130 \mathrm{~mA}$ and $R=1.7$. The rf-excitation was applied to damp the coherent instability.

\section{COOL EQUILIBRIUM}

The transverse beam-profiles were measured during EC. The beam-sizes were decreased by the EC, and saturated after cooling of $3 \mathrm{sec}$. In order to investigate the equilibrium beam-size depending on the intensity, the slow ionloss of a stacked ion-beam were sequencially measured after switching off the injection. In this measurement, the ion-beam is considered as the equilibrium after the cooling time constant of $\sim 3 \mathrm{sec}$.

Fig. 5 shows that the cross-section of the ion-beam was proportional to the intensity, so that the transverse density was kept constant at $0.9 \times 10^{7}$ ions $/ \mathrm{mm}^{2}$. The density cor- responds to the maximum betatron tune-shift of 0.03 [2] on the assumption of Gaussian distributions.

The behavior of $X Y \propto N$ can be related to some betatron resonance, and is also consistent with the Parkhomchuk's simulation result, taking into accout the diffusion losses caused by the electron beam [7].

At the low intensity of less than $\sim 10^{8}$ ions/ring, the ionbeam cross-section was restricted by the intra-beam scattering (IBS) and dependend on the $2 / 5$ 'th power of the intensity.

\section{SUMMARY}

In order to accumulate high intensity ion-beam, the coolstacking injection was conducted with electron cooling. The stacked intensity was increased with increasing the electron current and decreasing the diameter of the $\mathrm{e}^{-}$beam. Reducing of the $\mathrm{e}^{-}$-beam diameter minimized the slow beam-loss, while increasing of the electron current improved only the cooling rate. The mechanism of the slow beam-loss is not clearly understood, and further investigation is required. The diffusion process of the ion-beam inside the $\mathrm{e}^{-}$-beam can be one of the slow beam-loss source.

Coherent transverse instability was observed when ionand electron-beam density was high. The instability could be damped by applying rf-excitation and/or choosing the betatron tune far from the differential coupling resonance condition.

The limitation on the cooling corresponded to the ionbeam density of $\sim 0.9 \times 10^{7}$ ions $/ \mathrm{mm}^{2}$.

The authors thank the crews of the accelerator engineering cooporation (AEC) for their skillful operation of the HIMAC accelerator complex. This work has been performed as a part of Research Project with Heavy-Ion at NIRS-HIMAC.

\section{REFERENCES}

[1] Y. Hirao et al. , Nucl. Phys., A538(1992) 541c.

[2] K. Noda et al. , NIM A441(2000), 159.

[3] Y. Hashimoto et al. , NIM A527 (2004), 289.

[4] M. Beuterspacher et al. , Proc. of Ion Beam Cooling Toward the Crystalline Beam, Kyoto, 2001.

[5] T. Uesugi et al. , NIM A, to be published.

[6] K. Noda et al. , EPAC'04, Lucerne, Aug 2004.

[7] Parkhomchuk V. V., Proc. of ECOOL 01, 2001.

[8] V. V. Parkhomchuk and V. B. Reva, Jour. of Exper. and Theor. Phys., v.91, N5(2000), 975.

[9] P. R. Zenevich and A. E. Bolshakov, NIM A441 (2000), 36.

[10] A. Burov, NIM A441 (2000), 23.

[11] K. Noda et al. , NIM A492 (2002), 253. 